\title{
Evaluation of dosimetric impact of inter-application and intra-application variations in fractionated high-dose-rate intra-cavitary brachytherapy of cervical cancer
}

\author{
Tanvir Pasha, MBBS, MD, DNB!, Hanan Naniparuthayil Hassan, MBBS, MD², Nikhila Radhakrishna, MBBS, MD, DNB', \\ Muhammed Shafeeque N, MSc ${ }^{3}$, Ajin Shaji Varghese, MSc', Lokesh Viswanath, MBBS, MD', Nithin Bhaskar Valuvil, \\ MBBS, MD!, Sathiyan S, MSc, DipRP BARC, PhD',2, Govardhan H B, MBBS, MD, DNB', Ibrahim Khaleel, MBBS, MD!, \\ Siddanna Rudrappa Palled, MBBS, MD, DNB', Naveen Thimmaiah, MBBS, MDl
}

'Department of Radiation Oncology, Kidwai Memorial Institute of Oncology, Bengaluru, Karnataka, India, ${ }^{2}$ Department of Radiation Oncology, Bangalore Medical College and Research Institute, Bengaluru, Karnataka, India, 3Yenepoya Medical College Hospital, Deralakatte, Mangalore, Karnataka, India, ${ }^{4}$ Department of Radiation Physics, Kidwai Memorial Institute of Oncology, Bengaluru, Karnataka, India This work was conducted by all the authors at the Kidwai Memorial Institute of Oncology, Dr. M H Marigowda Rd., Bengaluru, 560029 Karnataka, India.

\begin{abstract}
Purpose: To evaluate feasibility and safety of execution of optimized intra-cavitary brachytherapy (ICBT) plan of first fraction in subsequent fractions in high-volume, low-resource centers.

Material and methods: This non-randomized prospective study included 30 cervical cancer patients, who underwent 4 fractions of high-dose-rate (HDR)-ICBT in 2 applications, one week apart, 2 fractions per application delivered on two consecutive days. Computed tomography (CT) simulation was done before each fraction, organs at risk (OARs) were contoured on all sets of CT images. Optimized plans were generated for each set of CT images and executed for the treatment. Test treatment plans were retrospectively generated by applying first treatment fraction's dwell times adjusted for decay, and dwell positions of the applicator for subsequent treatment fractions paired $t$-test was performed to analyze $\mathrm{D}_{2 \mathrm{cc}}$ dose variations of OARs among the paired sets of plans.

Results: Comparison between the plans showed daily plans provided lower $\mathrm{D}_{2 \mathrm{cc}}$ to OARs than test plans. In intraapplication plan comparison, there was a significant dose reduction to 2 cc sigmoid $(p=0.021)$ and bladder $(p=0.007)$ in daily plan. Mean $\mathrm{D}_{2 \mathrm{cc}}$ of optimized and unoptimized plans were $361.35 \pm 114.01$ and $411.70 \pm 152.73$ for sigmoid, and $511.23 \pm 85.47 \mathrm{cGy}$ and $553.57 \pm 111.23 \mathrm{cGy}$ for bladder, respectively. In inter-application, $\mathrm{D}_{2 \mathrm{cc}}$ rectum and sigmoid demonstrated a statistically significant dose variation $(p=0.002)$ and $(p=0.007)$, with mean $\mathrm{D}_{2 \mathrm{cc}}$ rectum of optimized and unoptimized plans being $401.06 \pm 83.53$ cGy and $452.46 \pm 123.97$ cGy, and of 2 cc sigmoid $340.84 \pm 117.90$ cGy and $387.79 \pm 141.36$ cGy, respectively.

Conclusions: Fractionated HDR brachytherapy amounts to significant variation in OAR doses if re-simulation and re-plan is not performed for every fraction and ICBT application. Therefore, plan of the day with optimization of the doses to target and OARs must be followed for each fraction.

Key words: cervical cancer, organ at risk, anatomical variation, intracavitary brachytherapy.

\section{Purpose}

Cervical uteri cancer is the second most common cancer among females in India. Sixty percent of cervical cancer patients present with locoregionally extensive clinical disease $[1,2]$. Brachytherapy is an integral part of locally advanced cervical cancer management, following external beam radiotherapy (EBRT) and concurrent chemotherapy [3, 4]. Currently, 2-dimensional X-ray-based brachytherapy planning has largely been replaced by 3-dimensional image-guided brachytherapy [5]. Magnetic resonance imaging (MRI)-based adaptive radiotherapy is the gold stan- 
dard of treatment in cervical intracavitary brachytherapy (ICBT) to reduce doses to organs at risk (OARs) to minimum, without compromising target coverage [6].

Several uncertainties prevail in image-guided brachytherapy (IGBT) planning, such as tumor regression between fractions, movement of OARs, and target in relation to applicator position and organ filling [7-9]. 3-dimensional computer tomography (CT)-based planning system, despite being available within several centers in developing nations, high patient load, and prevailing logistical reasons, prohibit its' maximum utilization. Therefore, many centers are often compelled to use first IGBT plan in subsequent fractions and applications.

The current study addressed a major problem faced in developing countries due to large patient volume, lower resources, and technological challenges in patient care. Our study aimed to evaluate the feasibility and safety of executing optimized intra-cavitary brachytherapy (ICBT) plan of first fraction in subsequent fractions and applications.

\section{Material and methods}

\section{Patient selection}

After obtaining an approval from institutional review board, patients with biopsy-proven squamous cell carcinoma (SCC), staged according to FIGO (International Federation of Gynecology and Obstetrics) 2018 [10] were selected from November 2017 to September 2019. 30 patients with good response to EBRT, whose residual disease was confined to medial parametrium and cervix were included in the current study.

\section{CT simulation and treatment overview}

All patients received pelvic EBRT of 45-50 Gray (Gy) in 25 fractions by 3D-CRT (3-dimensional conformal radiotherapy) technique. Concurrent chemotherapy of cisplatin $40 \mathrm{mg} / \mathrm{m}^{2}$ was administered weekly for 5-6 cycles. One week post-EBRT, patients underwent ICBT application using Manchester/Fletcher-Suit high-dose-rate (HDR) remote afterloading applicator under spinal anesthesia. Vaginal packing was done to fix the applicator in position and to displace the bladder and rectum away. All patients underwent two such applications, one week apart.

Computed tomography simulation was performed using a 16-slice helical CT scanner. Axial scan of $2.5 \mathrm{~mm}$ slice thickness was obtained 1-2 hours before each treatment. Strict bladder and rectal protocols were followed, with $50 \mathrm{cc}$ diluted iohexol contrast filled into the bladder via Foley's catheter, and $20 \mathrm{cc}$ contrast applied into the rectum.

Two sets of plans were created for each application on images obtained at 18-24 hours gap (intra-application planning). Inter-applications plan sets were created for a second application performed after a gap of 7 days. Further details of the plans are presented in the following section and Figure 1.

Organs at risk were delineated on all acquired images. High-risk clinical target volume (HR-CTV) was based on MRI imaging findings at diagnosis, clinical examination under anesthesia (EUA) during brachytherapy (BT), and radiological findings from $C T$ simulation scan, with the applicator in situ. A dose of 6.5 Gy was prescribed to point A at each fraction. This dose was further optimized to cover HR-CTV without compromising the dose to target

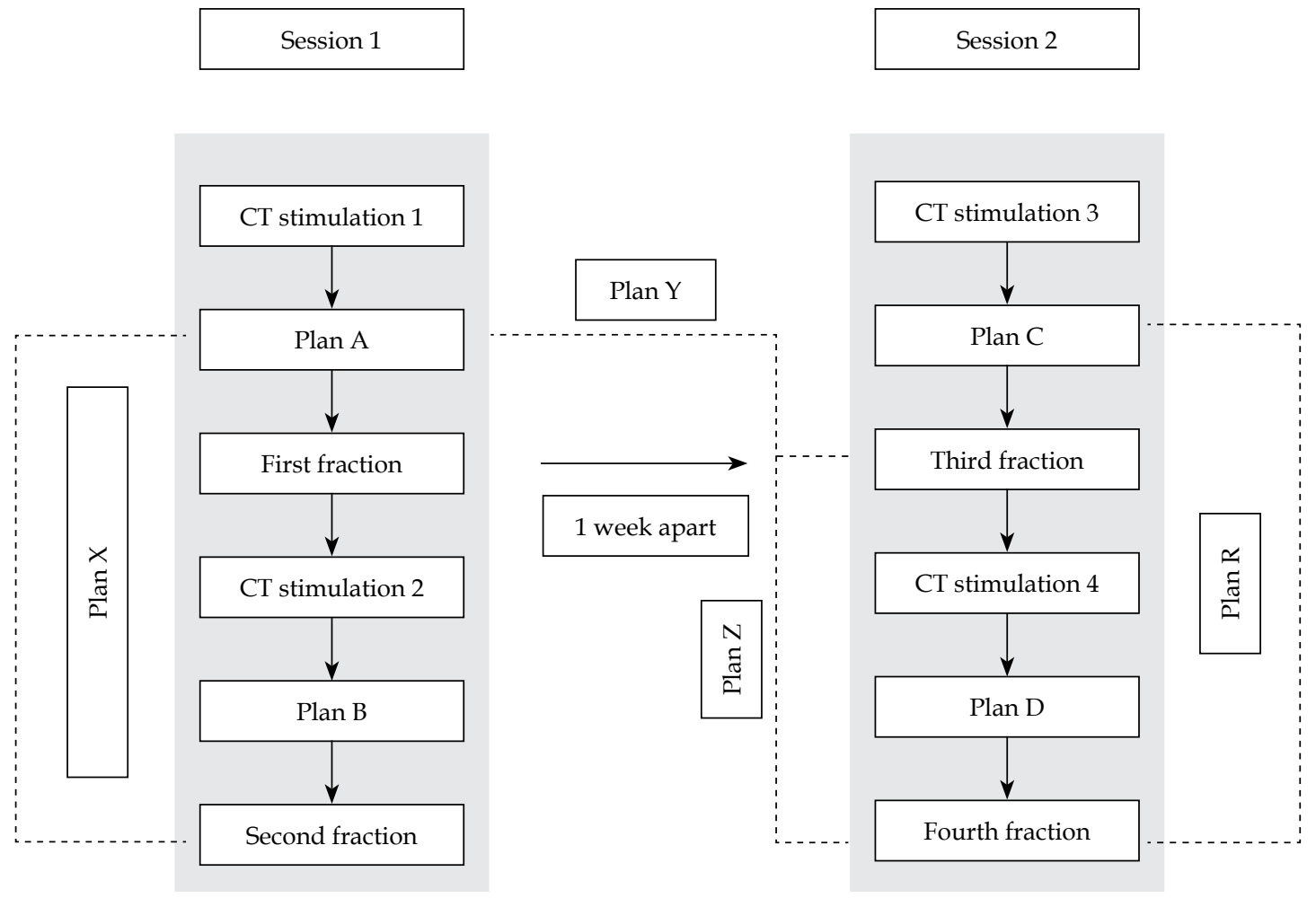

Fig. 1. Comparative plans 


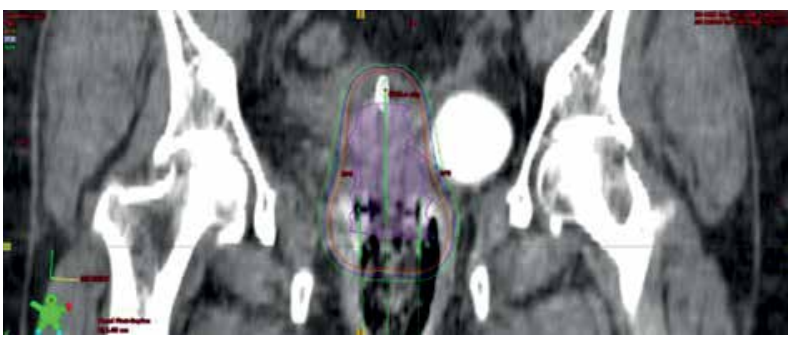

Fig. 2. Coronal section of CT scan of ICBT application with isodose distribution. Represented isodose levels are $100 \%$ dose (orange), $90 \%$ (blue), and $80 \%$ (green)

volume, while respecting OARs' tolerances (Figures 2-4). The accepted total dose limits as per our institutional protocol developed from the American Brachytherapy Society (ABS) and Indian Brachytherapy Society (IBS) guidelines, in equivalent 2 Gy dose $\left(\mathrm{EQD}_{2}\right)[11,12]$ were as follow:

a) Dose to point $A: E Q D_{2} \geq 75 G y$,

b) HR-CTV D ${ }_{90}: \mathrm{EQD}_{2} \geq 80 \mathrm{~Gy}$,

c) $\mathrm{D}_{2 \mathrm{cc}}$ rectum: $\mathrm{EQD}_{2} \leq 75 \mathrm{~Gy}$,

d) $\mathrm{D}_{2 \mathrm{cc}}$ bladder: $\mathrm{EQD}_{2} \leq 85 \mathrm{~Gy}$,

e) $\mathrm{D}_{2 \mathrm{cc}}$ sigmoid: $\mathrm{EQD}_{2} \leq 75 \mathrm{~Gy}$.

Brachytherapy was delivered using iridium-192 with Gamma Med plus HDR remote afterloading device (Varian Medical System Palo Alto, CA, USA). ICBT treatment was performed in two sessions; in each session, one fraction was delivered on the first day of application, and second fraction was given on the subsequent morning, leaving the applicator in situ overnight, with a time gap of 18 to 24 hours between two fractions.

\section{Comparative plans}

The following plans were generated in CT simulation scans (Figure 1):

- Plan A: An optimized plan in the first CT simulation.

- Plan B: A new optimized plan in the second CT simulation was taken before the second fraction (singleapplication).

- Plan C: A new plan optimized in the third CT simulation, taken before the third fraction (new application)

- Plan D: A new plan optimized in the fourth CT simulation, taken before the fourth fraction (same application as in plan $\mathrm{C}$ ).

- Plan X: A plan with the same dwell positions and timings (corrected for source decay) as the first fraction (plan A), and applied to the second CT simulation.

- Plan Y: A plan generated with the same dwell positions and timings (corrected for source decay) as the first fraction (plan A), and applied to the third CT simulation.

- Plan Z: A plan generated with the same dwell positions and timings (corrected for source decay) as the first fraction (plan A), and applied to the fourth CT simulation.

- Plan R: A plan generated with the same dwell positions and timings (corrected for source decay) as of the third fraction (plan C), and applied to the fourth CT simulation.

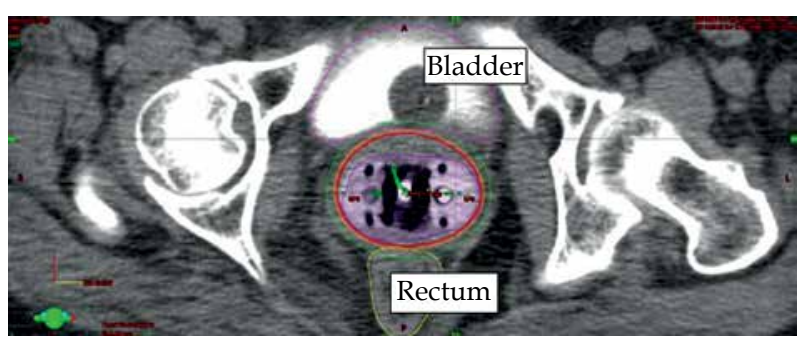

Fig. 3. Axial CT scan with applicator in place. Isodose levels of 650 cGy (orange) and 550 cGy (green) are presented

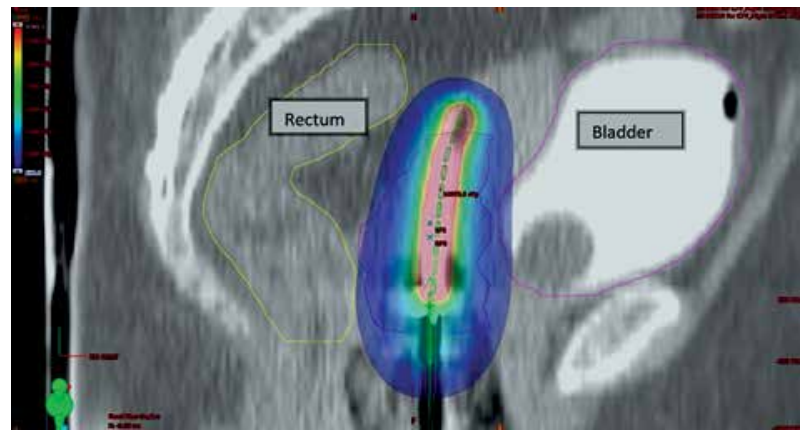

Fig. 4. Sagittal section of CT scan of ICBT application showing dose in color wash, blue shows prescribed dose 650 cGy

Intra-application variation: Dose variation between plan X and plan B; plan R and plan D; plan A and plan X.

Inter-application dose variation: Dose variation between plan Y and plan C; Dose variation between plan Z and plan D.

Dosimetric parameters were recorded for each fraction in point $\mathrm{A}$ doses, $\mathrm{D}_{2 \mathrm{cc}}$ bladder, rectum, and sigmoid.

\section{Statistical method}

Normality of data sets was analyzed and confirmed using Shapiro-Wilk test. Dosimetric parameters were expressed in terms of mean and standard deviation. Inter-application dosimetric variation and intra-application dosimetric variation were analyzed with paired $t$-test for comparison of means. Statistical tests were performed using Statistical Package for the Social Sciences (SPSS) 21 software. Any $p$-value of less than 0.05 was considered statistically significant.

\section{Results}

Patient characteristics at baseline are elaborated in Table 1. Among 30 patients, ten patients underwent all the planned four simulations, and the remaining $20 \mathrm{pa}-$ tients underwent three CT simulations (first three), owing to unavailability of a dedicated CT simulator in our department. Therefore, the plans were compared as described in Figure 1 for ten patients, with all four image sets. For the remaining 20 patients, intra-application variation was evaluated between the first and second fractions. Unfortunately, a fourth scan could not be obtained due to logistic limitations. Hence, plan $\mathrm{Z}$ and plan $\mathrm{R}$ could not be generated for comparison. 
Table 1. Patients' profile $(n=30)$

\begin{tabular}{llc} 
Age (years) & Range & $32-55$ \\
\cline { 2 - 3 } & Median & 46 \\
\hline \begin{tabular}{l} 
Stage (number of $\begin{array}{l}\text { patients) } \\
\text { par }\end{array}$ \\
\cline { 2 - 3 }
\end{tabular} & IIA & 0 \\
\cline { 2 - 3 } & IIIA & 3 \\
\cline { 2 - 3 } & III & 23 \\
\hline $\begin{array}{l}\text { Histologic type } \\
\text { (number of pa- } \\
\text { tients) }\end{array}$ & Squamous cell carcinoma & 30 \\
& &
\end{tabular}

Intra-application variation: In 40 sets of intra-application plans, 2 cc sigmoid dose was found to be significantly higher, if the first plan was executed on the second day without imaging and optimization. There was

Association between application and 2 cc SI

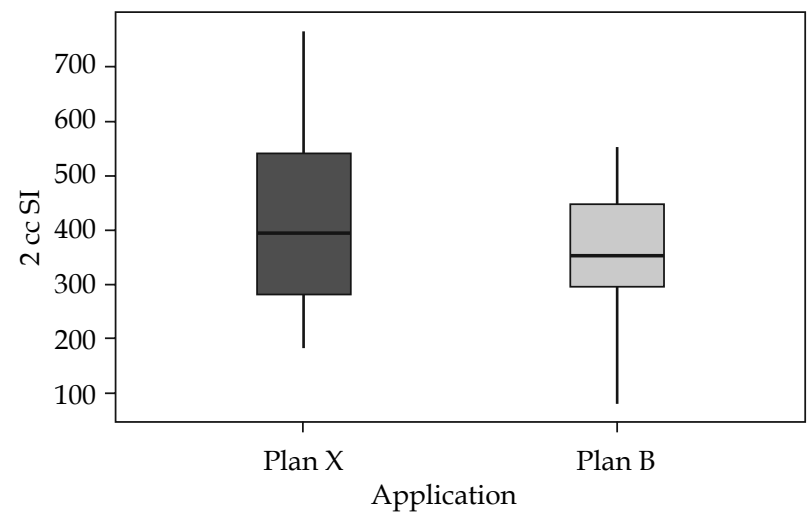

Fig. 5. Box-and-Whisker plot representation of intra-application variation plan $X$ and plan $B$ in sigmoid dose $(\mathrm{SI}=$ sigmoid $)$ a dose reduction and mean difference of $50 \pm 114$ cGy, with a $p$-value of 0.021 (Table 2). When a single plan was executed on consecutive days, doses to 2 cc sigmoid presented a wide range of variation compared to optimized plans, where variation was minimal (Figure 5). Also, there was a significant reduction in dose to $2 \mathrm{cc}$ bladder in plan A compared to plan $\mathrm{X}$, with a $p$-value of 0.007 with a mean $2 \mathrm{cc}$ doses of optimized and unoptimized plans being $511 \pm 85.5$ cGy and $553.6 \pm 111.2$ cGy, respectively (Table 3).

Inter-application variation: Doses to OARs, particularly 2 cc rectum and 2 cc sigmoid, showed significant reduction in the dose in new optimized plans. Mean difference of 2 cc rectal dose was 51.39 cGy ( \pm 85$)$, with a $p$-value of 0.002 , and 2 cc sigmoid was 46.9 cGy ( \pm 90$)$, with $p$-value of 0.007 , both $p$-values being statistically significant (Figures 6 and 7, Table 4).

All possible combinations of executed and applied plans for the 40 sets of scans in 10 patients were compared. The total mean $\mathrm{EQD}_{2}$ values were obtained as follows: 79 Gy for left point A (when single-plan dwell time and position applied in further scans), and 78 Gy for left point A (with imaging and optimization for each fraction). Similarly, the mean $\mathrm{EQD}_{2}$ of right point A with a single-plan was $78.5 \mathrm{~Gy}$, and with an individualized plan was 77.5 Gy. Hence, point A doses were more or less similar between optimized and non-optimized plans, but without statistical significance.

$\mathrm{EQD}_{2}$ of the bladder, rectum, and sigmoid exceeded tolerance limit in 4, 3, and 3 patients, respectively. 7 out of 30 patients had OARs' doses exceeding tolerance limits. Two patients presented with more than one organ receiving higher doses.

In Figures 5-7, Box-and-Whisker plot depicts the distribution of 2 cc OAR doses between two plans. The middle horizontal line represents the median 2 cc OARs' doses, and the upper and lower bounds indicate the $75^{\text {th }}$ and the $25^{\text {th }}$ centile, respectively.

Table 2. Intra-application variation between plan $X$ and plan $B$

\begin{tabular}{lccc} 
Dosimetric parameters & $\begin{array}{c}\text { Mean dose } \pm \text { SD (cGy) } \\
\text { Plan X }\end{array}$ & $\begin{array}{c}\text { Mean dose } \pm \text { SD (cGy) } \\
\text { Plan B }\end{array}$ & $\begin{array}{c}\text { Paired } t \text {-test } \\
p \text {-value }\end{array}$ \\
\hline Left point A & $538.15 \pm 104.96$ & $513.55 \pm 79.60$ & 1 \\
\hline Right point A & $535.30 \pm 94.32$ & $512.55 \pm 81.69$ & 1 \\
\hline$D_{2 c c}$ bladder & $553.57 \pm 111.23$ & $517.74 \pm 88.45$ & 0.068 \\
\hline$D_{2 c c}$ rectum & $429.55 \pm 111.03$ & $411.61 \pm 109.56$ & 1 \\
\hline$D_{2 c c}$ sigmoid & $411.69 \pm 152.73$ & $361.35 \pm 114.01$ & $0.021^{*}$
\end{tabular}

${ }^{*}$ statistically significant

Table 3. Intra-application variation between plan $A$ and plan $X$

\begin{tabular}{lccc} 
Dosimetric parameters & $\begin{array}{c}\text { Mean dose } \pm \text { SD (cGy) } \\
\text { Plan X }\end{array}$ & $\begin{array}{c}\text { Mean dose } \pm \text { SD (cGy) } \\
\text { Plan A }\end{array}$ & $\begin{array}{c}\text { Paired } t \text {-test } \\
p \text {-value }\end{array}$ \\
\hline Left point A & $538.15 \pm 104.96$ & $541.80 \pm 87.13$ & 1 \\
\hline Right point A & $535.30 \pm 94.32$ & $542.03 \pm 90.57$ & 1 \\
\hline$D_{2 c c}$ bladder & $553.57 \pm 111.23$ & $511.23 \pm 85.47$ & $0.007^{\star}$ \\
\hline$D_{2 c c}$ rectum & $429.55 \pm 111.03$ & $424.31 \pm 81.95$ & 1 \\
\hline$D_{2 c c}$ sigmoid & $411.69 \pm 152.73$ & $373.31 \pm 135.80$ & 0.224
\end{tabular}


Association between application and 2 cc R

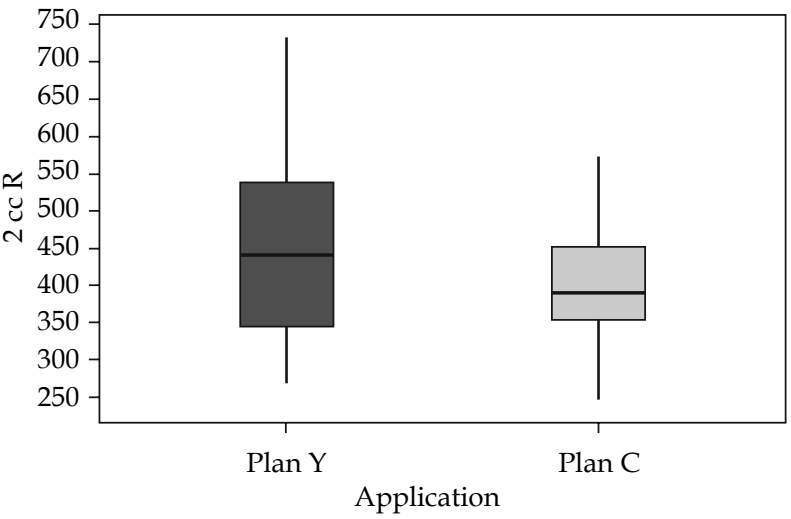

Fig. 6. Box-and-Whisker representation of inter-application dose variation of rectum plan $\mathrm{Y}$ and plan $\mathrm{C}(\mathrm{R}=$ rectum)
Association between application and 2 cc SI

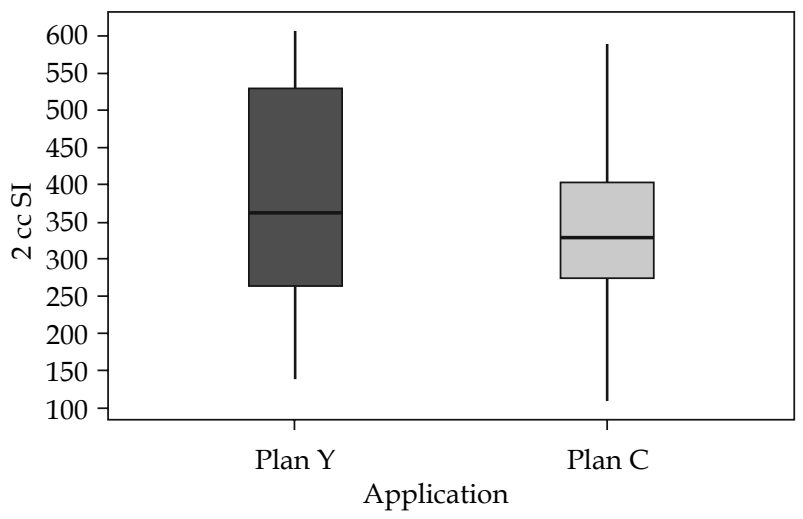

Fig. 7. Box-and-Whisker representation of inter-application dose variation of the sigmoid in plan $\mathrm{Y}$ and plan $\mathrm{C}$ $(\mathrm{SI}=$ sigmoid $)$

Table 4. Inter-application variation between plan $C$ and plan $Y$

\begin{tabular}{lccc} 
Dosimetric parameters & $\begin{array}{c}\text { Mean dose } \pm \text { SD (cGy) } \\
\text { Plan C }\end{array}$ & $\begin{array}{c}\text { Mean dose } \pm \text { SD (cGy) } \\
\text { Plan Y }\end{array}$ & $\begin{array}{c}\text { Paired } t \text {-test } \\
\text {-value }\end{array}$ \\
\hline Left point A & $536.61 \pm 82.48$ & $559.17 \pm 114.25$ & 0.273 \\
\hline Right point A & $541.23 \pm 82.95$ & $556.70 \pm 114.60$ & 0.436 \\
\hline$D_{2 c c}$ bladder & $500.00 \pm 89.40$ & $506.64 \pm 104.50$ & 0.678 \\
\hline$D_{2 c c}$ rectum & $401.06 \pm 83.53$ & $452.46 \pm 123.97$ & $0.002^{*}$ \\
\hline$D_{2 c c}$ sigmoid & $340.84 \pm 117.90$ & $387.79 \pm 141.36$ & $0.007^{*}$ \\
${ }^{*}$ statistically significant & & &
\end{tabular}

\section{Discussion}

MRI-based image-guided adaptive planning for each fraction is the existing optimal approach for cervical cancer HDR-BT treatment [11]. According to Pötter et al. [5], adaptive MRI planning has provided local control rates of $95-100 \%$ at three years, with ICBT in limited-stage disease (IB/IIB); 85-90\% with combined intra-cavitary + interstitial brachytherapy in advanced stages or poor response cervix cancer patients, with minimal treatment-related morbidity. However, CT scan is commonly used for target and OARs delineation due to its' wide availability and accessibility. Therefore, in the current study, CT-based contouring has been followed using the available guidelines [13, 14].

Grigsby et al. [15] observed a significant anatomical variation in gynecologic brachytherapy prescription points, because of changes in uterine axis, slippage of tandem and colpostat, patient movements, vaginal packing dislodgment, tumor regression between multiple fractions, and gaseous distention of the bowel. With all these uncertainties, our study attempted to investigate the feasibility and safety of the execution of previous day plan in subsequent fraction without any change in target coverage and OARs toxicity.

Al-Booz et al. [16] noted an increase in dose to the sigmoid in ICBT, when point A doses were not compromised. Similarly, the sigmoid colon was consistently observed to receive unknown variable doses in the current study if subsequent fractions were not imaged and planned.
Chakraborty et al. [17] fused two scan images and structure sets, and compared their plans with multiplanar reconstruction. $16 \%$ of their patients had their OARs tolerances exceeded, as the second fraction was not imaged. In our study, $\mathrm{EQD}_{2}$ of OARs exceeded in $23 \%$ of the patients (multiple patients had more than one organ crossing tolerance limits). Single-plan execution in subsequent applications by Kirisits et al. resulted in an average increase of $\mathrm{D}_{2 \mathrm{cc}}$ doses to bladder by $3.5 \mathrm{~Gy}$, rectum by $2.8 \mathrm{~Gy}$, and sigmoid by 5.8 Gy [18]. Additionally, a relative increase in D2 cc to the bladder by $9 \%$ was observed when a uniform bladder filling protocol was not followed [18]. Therefore, a constant bladder filling protocol was adopted in our study. However, variation in doses would have resulted from other uncertainties, such as alteration in volume of the vaginal packing due to vaginal or uterine secretions, and differential patient's hydration.

Mohammed et al. [19] evaluated the feasibility of applying a dose-optimized plan of ICBT and ISBT (interstitial brachytherapy) PDR (pulsed-dose-rate) brachytherapy of a first fraction to subsequent fraction. They intended to limit the number of scans and plans generated. Among 32 ICBT patients, dose delivered to $100 \%$ of target $\left(D_{100}\right)$ and volume of target receiving $100 \%$ of prescription dose $\left(\mathrm{V}_{100}\right)$ were significantly different. OARs doses were outside the tolerance limit for 5 out of 32 patients with a single-plan. Moreover, Chi et al. demonstrated a statistically significant increase in the absolute $D_{2 c c}$ dose to the bladder by $4.6 \mathrm{~Gy}$ and the rectum by $6.4 \mathrm{~Gy}$, when a sin- 
gle-ICBT plan was executed in subsequent fractions [20]. In the current study, if a single-plan was applied for subsequent fractions, $10 \%$ of patients $(3 / 30)$ would receive $\mathrm{EQD}_{2}$ to $2 \mathrm{cc}$ sigmoid and $2 \mathrm{cc}$ rectum beyond the level of tolerance. Even though it was not statistically significant, the total bladder dose in 3 of $30(10 \%)$ patients showed an increase of 2-8 Gy EQD 2 dose.

Centers with limited imaging access to brachytherapy may assume that it is safe to carry on brachytherapy insertions and treatment without imaging and re-planning for each insertion. However, the current study emphasizes that such a practice would be unsafe due to an increase in OARs doses. This, in turn, may contribute to several acute and late radiation toxicities. Therefore, it is suggested that centers with logistic challenges should explore other possibilities, including prior coordination with a radiology department or obtaining dedicated time slots on imaging machines for ICBT cases.

Drawbacks of the current study include a small sample size and the inability to acquire the fourth CT scan for 20 patients, owing to the lack of a dedicated CT simulator in our department. MRI at BT with applicator in situ, or even before BT, could not be obtained due to availability of a single-MRI unit designated for the entire hospital, and non-availability of MR-compatible applicators. Applicator geometry, organ deformations, and target response are few confounding factors, which could affect dose distributions. Further studies are required to analyze the impact of these factors upon inter-application and intra-application dose variations.

Dedicated CT simulators and MR-compatible applicators have recently been acquired at our Institute. Based on the outcomes of the current study, we have adopted brachytherapy planning prior to each fraction.

\section{Conclusions}

Fractionated HDR brachytherapy amounts to significant variation in OARs doses if re-simulation and re-plan is not performed for every fraction and ICBT application. Therefore, plan of the day with optimization of the doses to target and OARs must be followed for each fraction.

\section{Disclosure}

The authors report no conflict of interest.

\section{References}

1. Bray F, Ferlay J, Soerjomataram I et al. Global cancer statistics 2018: GLOBOCAN estimates of incidence and mortality worldwide for 36 cancers in 185 countries. CA Cancer J Clin 2018; 68: 394-424. Erratum in: CA Cancer J Clin 2020; 70: 313.

2. Sreedevi A, Javed R, Dinesh A. Epidemiology of cervical cancer with special focus on India. Int J Womens Health 2015; 7: 405-414.

3. Nag S, Erickson B, Thomadsen B et al. The American Brachytherapy Society recommendations for high-dose-rate brachytherapy for carcinoma of the cervix. Int J Radiat Oncol Biol Phys 2000; 48: 201-211.

4. Nori D, Dasari N, Allbright RM. Gynecologic brachytherapy I: Proper incorporation of brachytherapy into the current multimodality management of carcinoma of the cervix. Semin Radiat Oncol 2002; 12: 40-52.
5. Pötter R, Georg P, Dimopoulos JC et al. Clinical outcome of protocol-based image (MRI) guided adaptive brachytherapy combined with 3D conformal radiotherapy with or without chemotherapy in patients with locally advanced cervical cancer. Radiother Oncol 2011; 100: 116-123.

6. Dimopoulos JC, Petrow P, Tanderup K et al. Recommendations from Gynaecological (GYN) GEC-ESTRO Working Group (IV): Basic principles and parameters for MR imaging within the frame of image based adaptive cervix cancer brachytherapy. Radiother Oncol 2012; 103: 113-122.

7. Lang S, Nesvacil N, Kirisits C et al. Uncertainty analysis for 3D image-based cervix cancer brachytherapy by repetitive MR imaging: assessment of DVH-variations between two HDR fractions within one applicator insertion and their clinical relevance. Radiother Oncol 2013; 107: 26-31.

8. Hellebust TP, Dale E, Skjønsberg A et al. Inter fraction variations in rectum and bladder volumes and dose distributions during high dose rate brachytherapy treatment of the uterine cervix investigated by repetitive CT-examinations. Radiother Oncol 2001; 60: 273-280.

9. De Leeuw AA, Moerland MA, Nomden C et al. Applicator reconstruction and applicator shifts in 3D MR-based PDR brachytherapy of cervical cancer. Radiother Oncol 2009; 93: 341-346.

10. Bhatla N, Aoki D, Sharma DN et al. Cancer of the cervix uteri. Int J Gynaecol Obstet 2018; 143 Suppl 2: 22-36.

11. Viswanathan AN, Beriwal S, De Los Santos JF et al. American Brachytherapy Society. American Brachytherapy Society consensus guidelines for locally advanced carcinoma of the cervix. Part II: high-dose-rate brachytherapy. Brachytherapy 2012; 11: 47-52.

12. Mahantshetty U, Gudi S, Singh R et al. Indian Brachytherapy Society Guidelines for radiotherapeutic management of cervical cancer with special emphasis on high-dose-rate brachytherapy. J Contemp Brachytherapy 2019; 11: 293-306.

13. Viswanathan AN, Erickson B, Gaffney DK et al. Comparison and consensus guidelines for delineation of clinical target volume for CT- and MR-based brachytherapy in locally advanced cervical cancer. Int I Radiat Oncol Biol Phys 2014; 90: 320-328.

14. Mahantshetty U, Potter R, Beriwal $S$ et al. IBS-GEC ESTRO-ABS recommendations for CT based contouring in image-guided adaptive brachytherapy for cervical cancer. Radiother Oncol 2021; 160: 273-284.

15. Grigsby PW, Georgiou A, Williamson JF et al. Anatomic variation of gynecologic brachytherapy prescription points. Int J Radiat Oncol Biol Phys 1993; 27: 725-729.

16. Al-Booz H, Boiangiu I, Appleby $\mathrm{H}$ et al. Sigmoid colon is an unexpected organ at risk in brachytherapy for cervix cancer. J Egypt Natl Cancer Inst 2006; 18: 156-160.

17. Chakraborty S, Patel FD, Patil VM et al. Magnitude and implications of interfraction variations in organ doses during high dose rate brachytherapy of cervix cancer: A CT based planning study. ISRN Oncol 2014; 2014: 687365.

18. Kirisits C, Lang S, Dimopoulos J et al. Uncertainties when using only one MRI-based treatment plan for subsequent highdose-rate tandem and ring applications in brachytherapy of cervix cancer. Radiother Oncol 2006; 81: 269-275.

19. Mohamed S, Nielsen SK, Fokdal LU et al. Feasibility of applying a single treatment plan for both fractions in PDR image guided brachytherapy in cervix cancer. Radiother Oncol 2013; 107: 32-38.

20. Chi A, Gao M, Sinacore J et al. Single versus customized treatment planning for image-guided high-dose-rate brachytherapy for cervical cancer: dosimetric comparison and predicting factor for organs at risk overdose with single plan approach. Int J Radiat Oncol Biol Phys 2009; 75: 309-314. 